\title{
Complete genome sequence of Methanoculleus marisnigri Romesser et al. 1981 type strain JR1
}

\author{
lain J. Anderson ${ }^{1 *}$, Magdalena Sieprawska-Lupa², Alla Lapidus ${ }^{1}$, Matt Nolan ${ }^{1}$, Alex Copel- \\ and1, Tijana Glavina Del Rio', Hope Tice1, Eileen Dalin', Kerrie Barry', Elizabeth Saund- \\ ers $^{1,3}$, Cliff Han ${ }^{1,3}$, Thomas Brettin ${ }^{1,3}$, John C. Detter ${ }^{1,3}$, David Bruce ${ }^{1,3}$, Natalia Mikhailova ${ }^{1}$, \\ Sam Pitluck ${ }^{1}$, Loren Hauser ${ }^{1,4}$, Miriam Land ${ }^{1,4}$, Susan Lucas ${ }^{1}$, Paul Richardson ${ }^{1}$, William B. \\ Whitman², and Nikos C. Kyrpides ${ }^{1}$ \\ 1Joint Genome Institute, 2800 Mitchell Drive, Walnut Creek, California, USA \\ ${ }^{2}$ Microbiology Department, University of Georgia, Athens, Georgia, USA \\ ${ }^{3}$ Los Alamos National Laboratory, Bioscience Division, Los Alamos, New Mexico, USA \\ ${ }^{4}$ Oak Ridge National Laboratory, Oak Ridge, Tennessee, USA
}

*Corresponding author: lain Anderson

Keywords: Archaea, methanogen, Methanomicrobiales

Methanoculleus marisnigri Romesser et al. 1981 is a methanogen belonging to the order Methanomicrobiales within the archaeal phylum Euryarchaeota. The type strain, JR1, was isolated from anoxic sediments of the Black Sea. M. marisnigri is of phylogenetic interest because at the time the sequencing project began only one genome had previously been sequenced from the order Methanomicrobiales. We report here the complete genome sequence of M. marisnigri type strain JR1 and its annotation. This is part of a Joint Genome Institute 2006 Community Sequencing Program to sequence genomes of diverse Archaea.

\section{Introduction}

Methanoculleus marisnigri is a methanogen belonging to the order Methanomicrobiales, and strain JR1 is the type strain of this species. When it was first isolated, this organism was named $\mathrm{Me}$ thanogenium marisnigri [1], but then later it was transferred to the genus Methanoculleus [2]. The type strain was isolated from sediment of the Black Sea, while another strain was isolated from an anaerobic digestor [2]. Other species of Methanoculleus have been isolated from different types of anaerobic digestors and marine and freshwater sediments (reviewed in [3]).

Methanogens have been divided into two groups known as Class I and Class II based on phylogeny [4]. Class I includes the orders Methanococcales, Methanobacteriales, and Methanopyrales, which use $\mathrm{H}_{2} / \mathrm{CO}_{2}$ or formate as substrates for methanogenesis, although some can also use alcohols as electron donors. Class II includes the orders $\mathrm{Me}$ thanosarcinales and Methanomicrobiales. Some of the Methanosarcinales are capable of using various methyl compounds as substrates for methanogenesis including acetate, methylamines, and me- thanol, but Methanomicrobiales are restricted to the same substrates as the Class I methanogens [3]. Therefore Methanomicrobiales are phylogenetically closer to Methanosarcinales but physiologically more similar to Class I methanogens, making them an interesting target for genome sequencing. In a 2006 Community Sequencing Program (CSP) project, we proposed sequencing two members of the order Methanomicrobiales: M. marisnigri and Methanocorpusculum labreanum. Previously only one genome was available from this order, that of Methanospirillum hungatei. M. marisnigri and $M$. labreanum are phylogenetically distant from each other and from M. hungatei (Figure 1), and they represent the three phylogenetic families within the order Methanomicrobiales. We report here the sequence and annotation of $M$. marisnigri type strain JR1.

\section{Classification and features}

Methanoculleus marisnigri JR1 was isolated from Black Sea sediment at a depth of $0.5-20 \mathrm{~cm}$. The enrichment medium consisted of $30 \%$ distilled 
water and $70 \%$ sea water with the addition of acetate, formate, trypticase, yeast extract, vitamin solution, trace mineral solution, and volatile fatty acid solution [1]. Cells were maintained in serum vials under an atmosphere of $80 \% \mathrm{H}_{2}$ and $20 \%$ $\mathrm{CO}_{2}$ by a modification of the Hungate technique [1]. The physiological characteristics of $M$. marisnigri were described as follows [1]. The cells were irregular cocci with peritrichous flagella. The cell wall was composed of glycoprotein and lacked peptidoglycan. The optimal growth temperature was $20-25^{\circ} \mathrm{C}$ with growth observed between 15 and $45^{\circ} \mathrm{C}$. The optimal $\mathrm{pH}$ for growth was 6.4 with a range of 6.0-7.5. The optimal salt concentration for growth was around $0.1 \mathrm{M} \mathrm{NaCl}$, and growth was observed between 0.0 and $0.7 \mathrm{M} \mathrm{NaCl}$. Neither acetate nor yeast extract was stimulatory for growth. Trypticase was required, and it could not be replaced by Casamino acids or other peptide mixtures. Coenzyme $\mathrm{M}$ and Coenzyme $\mathrm{F}_{420}$ were both detected in $M$. marisnigri. Growth was observed with $\mathrm{H}_{2} / \mathrm{CO}_{2}$ or formate but not with acetate or methanol. M. marisnigri was subsequently shown to grow with secondary alcohols as the electron donor for methanogenesis [6]. The physiological and morphological features of $M$. marisnigri are presented in (Table 1).

\section{Genome sequencing information} Genome project history

Methanoculleus marisnigri was selected for sequencing based upon its phylogenetic position relative to other methanogens of the order Methanomicrobiales. It is part of a Joint Genome Institute 2006 Community Sequencing Program project that included six archaeal genomes selected for their phylogenetic diversity. A summary of the project information is shown in Table 2. The complete genome sequence was finished in February, 2007. The GenBank accession number for the project is CP000562. The genome project is listed in the Genomes OnLine Database (GOLD) [18] as project Gc00512. Sequencing was carried out at the Joint Genome Institute (JGI) Production Genomics Facility (PGF) in Walnut Creek, California. Quality assurance using Phred $[19,20]$ was done by JGI-Stanford. Finishing was done by JGI-Los Alamos National Laboratory (LANL). Annotation was done by JGI-Oak Ridge National Laboratory (ORNL) and by JGI-PGF.

\section{Growth conditions and DNA isolation}

The methods for DNA isolation, genome sequencing and assembly for this genome have previously been published [21].

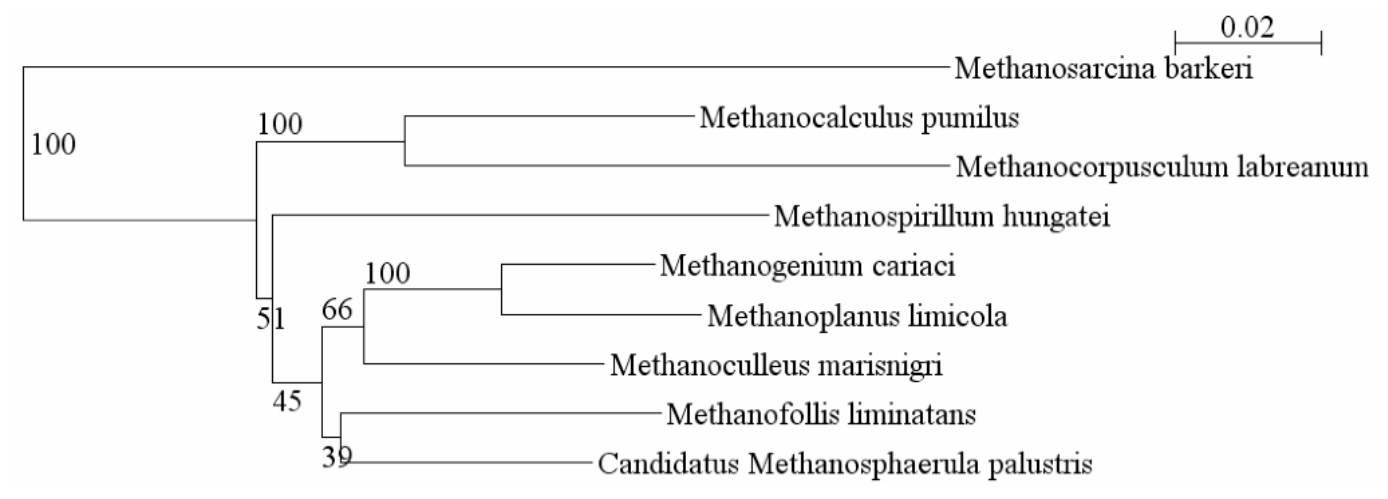

Figure 1. Phylogenetic tree of selected Methanomicrobiales showing the distance between the three organisms for which complete genomes are available - Methanospirillum hungatei, Methanocorpusculum labreanum, and Methanoculleus marisnigri. The tree uses 16S ribosomal RNA sequences aligned within the Ribosomal Database Project (RDP), and the tree was constructed with the RDP Tree Builder [5]. Methanosarcina barkeri was used as the outgroup. The numbers on the branches represent bootstrap values based on 100 replicates. 
Table 1. Classification and general features of M. marisnigri JR1 according to the MIGS recommendations [7].

\begin{tabular}{|c|c|c|c|}
\hline MIGS ID & Property & Term & Evidence Code \\
\hline & Current classification & Domain Archaea & TAS $[8-10$ \\
\hline & & Phylum Euryarchaeota & TAS $[11,12]$ \\
\hline & & Class "Methanomicrobia" & TAS [13] \\
\hline & & Order Methanomicrobiales & TAS [14] \\
\hline & & Family Methanomicrobiaceae & TAS [14] \\
\hline & & Genus Methanoculleus & TAS [2] \\
\hline & & Species Methanoculleus marisnigri & TAS [2] \\
\hline & Gram stain & negative & \\
\hline & Cell shape & irregular coccus & TAS [1] \\
\hline & Motility & peritrichous flagella & TAS [1] \\
\hline & Sporulation & nonsporulating & NAS \\
\hline & Temperature range & $15-45^{\circ} \mathrm{C}$ & TAS [1] \\
\hline & Optimum temperature & $20-25^{\circ} \mathrm{C}$ & TAS [1] \\
\hline MIGS-6.3 & Salinity & $0.0-0.7 \mathrm{M} \mathrm{NaCl}$ & TAS [1] \\
\hline \multirow[t]{3}{*}{ MIGS-22 } & Oxygen requirement & anaerobe & TAS [1] \\
\hline & Carbon source & $\mathrm{CO}_{2}$ & NAS \\
\hline & Energy source & $\mathrm{H}_{2} / \mathrm{CO}_{2}$, formate, secondary alcohols & TAS $[1,6]$ \\
\hline MIGS-6 & Habitat & sediment, anaerobic digestors & TAS $[1,2]$ \\
\hline MIGS-15 & Biotic relationship & free-living & TAS [1] \\
\hline \multirow[t]{3}{*}{ MIGS-14 } & Pathogenicity & none & NAS \\
\hline & Biosafety level & 1 & NAS \\
\hline & Isolation & sediment & TAS [1] \\
\hline MIGS-4 & Geographic location & Black Sea & TAS [1] \\
\hline MIGS-5 & Isolation time & 1979 & TAS [1] \\
\hline MIGS-4.1 & Latitude-longitude & not reported & \\
\hline MIGS-4.2 & & & \\
\hline MIGS-4.3 & Depth & $0.5-20 \mathrm{~cm}$ & TAS [1] \\
\hline MIGS-4.4 & Altitude & not applicable & \\
\hline
\end{tabular}

Evidence codes - IDA: Inferred from Direct Assay (first time in publication); TAS: Traceable Author Statement (i.e., a direct report exists in the literature); NAS: Non-traceable Author Statement (i.e., not directly observed for the living, isolated sample, but based on a generally accepted property for the species, or anecdotal evidence). These evidence codes are from the Gene Ontology project [15]. If the evidence code is IDA, then the property was directly observed for a living isolate by one of the authors or another expert mentioned in the acknowledgements.

Table 2. Genome sequencing project information

\begin{tabular}{lll}
\hline MIGS ID & Characteristic & Details \\
\hline MIGS-28 & Libraries used & $3 \mathrm{~kb}, 6 \mathrm{~kb}$ and 40kb (fosmid) \\
MIGS-29 & Sequencing platform & Applied Biosystems 3730 \\
MIGS-31.2 & Sequencing coverage & $11 \times$ \\
MIGS-31 & Finishing quality & Finished \\
& Sequencing quality & less than one error per 50kb \\
MIGS-30 & Assembler & Phrap \\
MIGS-32 & Gene calling method & CRITICA [16], Glimmer [17] \\
& GenBank ID & CPO00562 \\
& GenBank date of release & October 17, 2007 \\
& GOLD ID & Gc00512 \\
& NCBI project ID & 16330 \\
& IMG Taxon ID & 640069318 \\
MIGS-13 & Source material identifier & ATCC 35101 \\
& Project relevance & phylogenetic diversity \\
\hline
\end{tabular}




\section{Genome properties}

The genome of $M$. marisnigri JR1 consists of a single circular chromosome (Figure 2 and Table 3). In comparison with other methanogens, the genome size of $2.48 \mathrm{Mbp}$ is larger than those of Class I methanogens, which tend to be $1.6-1.8 \mathrm{Mbp}$, but smaller than the genomes of Methanosarcina species and Methanospirillum hungatei, which range between 3.5 and $5.8 \mathrm{Mbp}$. The $\mathrm{G}+\mathrm{C}$ percentage of M. marisnigri is $62.1 \%$, the highest among sequenced methanogens. The genome contains 2,560 genes of which 2,506 are protein-coding genes and the remaining 54 are RNA genes. There were only 17 pseudogenes identified, constituting $0.68 \%$ of the total genes. In total, 1633 proteincoding genes $(65.2 \%)$ were assigned a function, with the remaining annotated as hypothetical proteins. The percentage of genes with signal peptides $(14.0 \%)$ is quite high compared to other methanogens, although several methanogens have similar percentages. The properties and statistics of the genome are summarized in Table 3 and genes belonging to COG functional categories are listed in Table 4.

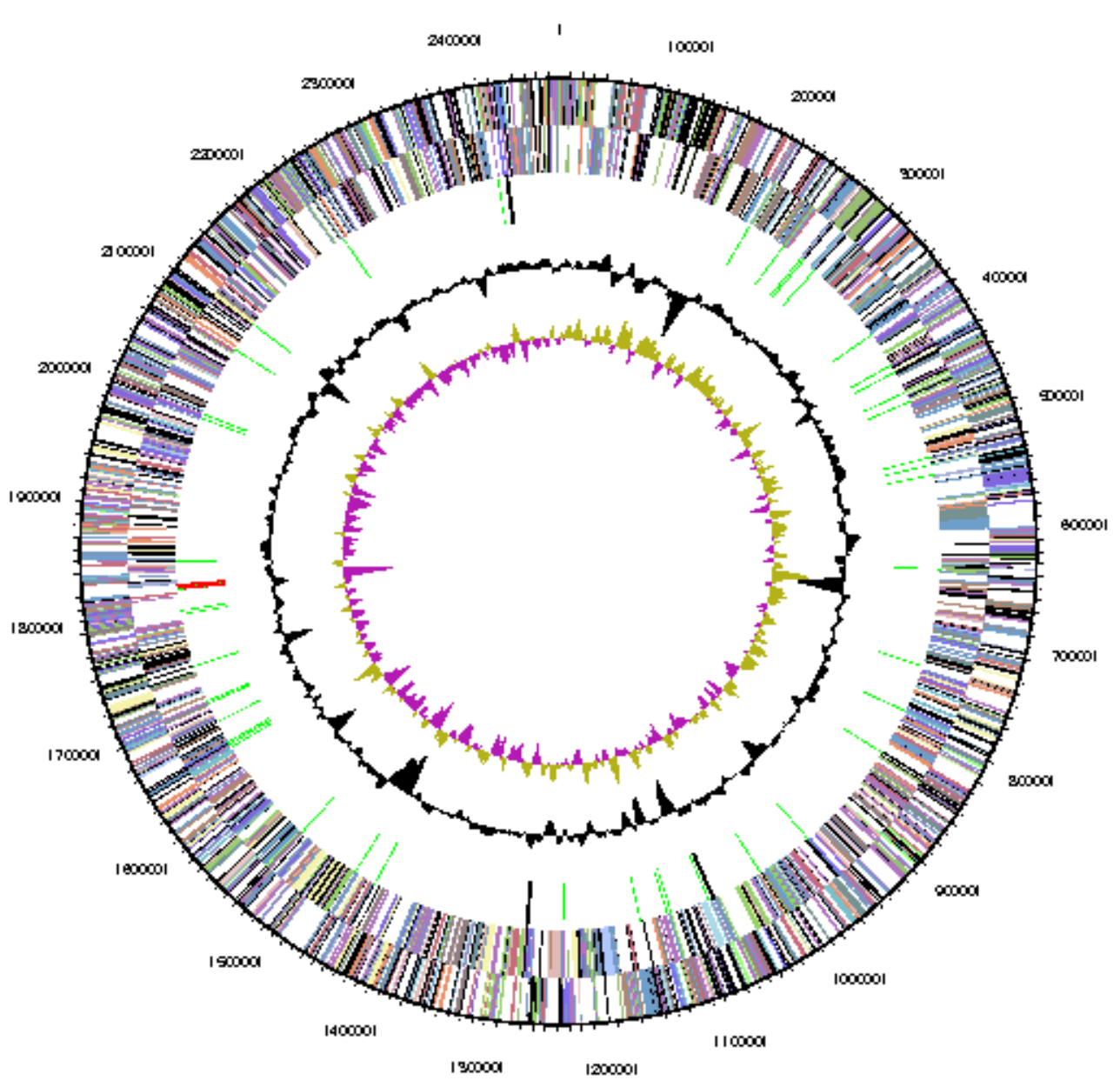

Figure 2. Graphical circular map of the chromosome. From outside to the center: Genes on forward strand (colored by COG categories), Genes on reverse strand (colored by COG categories), RNA genes (tRNAs green, rRNAs red, other RNAs black), GC content, GC skew. 
Table 3. Genome statistics

\begin{tabular}{lrr}
\hline Genome characteristic & Value & \% of total \\
\hline Genome size (bp) & $2,478,10$ & $100.00 \%$ \\
& 1 & \\
DNA coding region (bp) & $2,181,39$ & $88.0 \%$ \\
& 3 & \\
DNA G+C content (bp) & $1,537,98$ & $62.1 \%$ \\
& 1 & \\
Number of replicons & 1 & \\
Extrachromosomal elements & 0 & \\
Total genes & 2560 & $100.00 \%$ \\
RNA genes & 54 & $2.1 \%$ \\
rRNA operons & 1 & \\
Protein-coding genes & 2506 & $97.9 \%$ \\
Pseudogenes & 17 & $0.7 \%$ \\
Genes with function prediction & 1633 & $65.2 \%$ \\
Genes in paralog clusters & 1230 & $49.1 \%$ \\
Genes assigned to COGs & 1985 & $79.2 \%$ \\
Genes assigned Pfam domains & 1790 & $71.4 \%$ \\
Genes with signal peptides & 352 & $14.0 \%$ \\
Genes with transmembrane helices & 595 & $23.7 \%$ \\
CRISPR repeats & 0 & \\
\hline
\end{tabular}

Table 4. Numbers of genes associated with general COG functional categories.

\begin{tabular}{lrrl}
\hline Code & Value & \% age & Description \\
\hline E & 139 & 5.5 & Amino acid transport and metabolism \\
G & 77 & 3.1 & Carbohydrate transport and metabolism \\
D & 17 & 0.7 & Cell cycle control, cell division, chromosome partitioning \\
N & 23 & 0.9 & Cell motility \\
M & 104 & 4.2 & Cell wall/membrane/envelope biogenesis \\
B & 5 & 0.2 & Chromatin structure and dynamics \\
H & 152 & 6.1 & Coenzyme transport and metabolism \\
Z & 0 & 0.0 & Cytoskeleton \\
V & 23 & 0.9 & Defense mechanisms \\
C & 174 & 6.9 & Energy production and conversion \\
W & 0 & 0.0 & Extracellular structures \\
S & 255 & 10.2 & Function unknown \\
R & 286 & 11.4 & General function prediction only \\
P & 94 & 3.8 & Inorganic ion transport and metabolism \\
U & 22 & 0.9 & Intracellular trafficking, secretion, and vesicular transport \\
I & 30 & 1.2 & Lipid transport and metabolism \\
Y & 0 & 0.0 & Nuclear structure \\
F & 63 & 2.5 & Nucleotide transport and metabolism \\
O & 84 & 3.4 & Posttranslational modification, protein turnover, chape- \\
& & & rones \\
A & 1 & 0.0 & RNA processing and modification \\
L & 84 & 3.4 & Replication, recombination and repair \\
Q & 15 & 0.6 & Secondary metabolites biosynthesis, transport and catabol- \\
& & & ism \\
T & 87 & 3.5 & Signal transduction mechanisms \\
K & 97 & 3.9 & Transcription \\
J & 153 & 6.1 & Translation, ribosomal structure and biogenesis \\
- & 521 & 20.8 & Not in COGs \\
\hline & & &
\end{tabular}




\section{Insights from the genome sequence}

The genome sequence of $M$. marisnigri JR1 shows some similarities to Class I methanogens and some to Methanosarcinales but also has some unique features. In common with Class I methanogens, $M$. marisnigri uses a partial reductive TCA cycle to synthesize 2-oxoglutarate, and it has the Eha membrane-bound hydrogenase. Similar to Methanosarcinales, M. marisnigri has the Ech membrane-bound hydrogenase. A unique feature of $M$. marisnigri and the other Methanomicrobiales is the presence of anti- and anti-anti-sigma factors, which is surprising as Archaea do not use sigma factors. These anti- and anti-anti-sigma factors must have developed a new function in the Archaea. Phylogenetic analysis of methanogenesis and cofactor biosynthesis enzymes suggests that Methanomicrobiales form a group distinct from other methanogens, and therefore methanogens can be split in to three classes [21].

There are also differences among the Methanomicrobiales. For instance, M. marisnigri is the only one of the three to have the $\mathrm{F}_{420}$-nonreducing hy-

\section{References}

1. Romesser JA, Wolfe RS, Mayer F, Spiess E, Walther-Mauruschat A. Methanogenium, a new genus of marine methanogenic bacteria, and characterization of Methanogenium cariaci sp. nov. and Methanogenium marisnigri sp. nov. Arch Microbiol 1979; 121:147-153. doi:10.1007/BF00689979

2. Maestrojuán GM, Boone DR, Xun L, Mah RA, Zhang L. Transfer of Methanogenium bourgense, Methanogenium marisnigri, Methanogenium olentangyi, and Methanogenium thermophilicum to the genus Methanoculleus gen. nov., emendation of Methanoculleus marisnigri and Methanogenium, and description of new strains of Methanoculleus bourgense and Methanoculleus marisnigri. Int J Syst Bacteriol 1990; 40:117-122.

3. Garcia JL, Ollivier B, Whitman WB. The order Methanomicrobiales. Prokaryotes 2006; 3:208230. doi:10.1007/0-387-30743-5 10

4. Bapteste É, Brochier C, Boucher Y. Higher-level classification of the Archaea: evolution of methanogenesis and methanogens. Archaea 2005; 1:353-363. PubMed doi:10.1155/2005/859728

5. Cole JR, Wang Q, Cardenas E, Fish J, Chai B, Farris RJ, Kulam-Syed-Mohideen AS, McGarrell DM, Marsh T, Garrity GM, et al. The ribosomal data- drogenase, and it is the only one of the three to lack the 14-subunit Mbh membrane-bound hydrogenase. This has implications for the mechanism of methanogenesis: $M$. marisnigri may couple Coenzyme M-Coenzyme $B$ heterodisulfide reduction to the first step of methanogenesis in the cytoplasm, similar to Class I methanogens [35], while the other Methanomicrobiales may couple heterodisulfide reduction to generation of a membrane ion gradient [21].

\section{Acknowledgments}

This work was performed under the auspices of the US Department of Energy's Office of Science, Biological and Environmental Research Program, and by the University of California, Lawrence Berkeley National Laboratory under Contract No. DE-AC02-05CH11231, Lawrence Livermore National Laboratory under Contract No. DEAC52-07NA27344, and Los Alamos National Laboratory under Contract No. DE-AC02-06NA25396. M. L. was supported by the Department of Energy under contract DE-AC05-000R22725. M. S.-L., and W. B. W. were supported by DOE contract number DE-FG02-97ER20269.

base project: improved alignments and new tools for rRNA analysis. Nucleic Acids Res 2009; 37:D141-D145. PubMed doi:10.1093/nar/gkn879

6. Zellner G, Winter J. Secondary alcohols as hydrogen donors for $\mathrm{CO}_{2}$-reduction by methanogens. FEMS Microbiol Lett 1987; 44:323-328.

7. Field D, Garrity G, Gray T, Morrison N, Selengut J, Sterk P, Tatusova T, Thomson N, Allen MJ, Angiuoli SV, et al. The minimum information about a genome sequence (MIGS) specification. Nat Biotechnol 2008; 26:541-547. PubMed doi:10.1038/nbt1360

8. Woese CR, Kandler O, Wheelis ML. Towards a natural system of organisms: proposal for the domains Archaea, Bacteria, and Eucarya. Proc Natl Acad Sci USA 1990; 87:4576-4579. doi:10.1073/pnas.87.12.4576

9. Balch WE, Fox GE, Magrum LJ, Woese CR, Wolfe RS. Methanogens: reevaluation of a unique biological group. Microbiol Rev 1979; 43: 260-296. PubMed

10. List Editor. Validation List no. 6. Validation of the publication of new names and new combinations previously effectively published outside the IJSB. Int I Syst Bacteriol 1981; 31: 215-218. 
Anderson, et al.

11. Garrity GM, Holt JG. Phylum All. Euryarchaeota phy. nov. In Bergey's Manual of Systematic Bacteriology, vol. 1. 2nd ed. Edited by: Garrity, GM, Boone, DR and Castenholz, RW. Springer, New York; 2001:211-355.

12. List Editor. Validation of publication of new names and new combinations previously effectively published outside the IJSEM. Validation List no. 85. Int J Syst Evol Microbiol 2002; 52: 685690. PubMed doi:10.1099/ijs.0.02358-0

13. Garrity GM, Bell JA, Lilburn T. The revised road map to the manual. In: Brenner, DJ, Kreig, NR, Staley, JT Eds. 2009. Bergey's Manual of Systematic Bacteriology, $2^{\text {nd }} E d$. Vol 2 The Proteobacteria Part A Introductory Essays. 2005 pp 159-220

14. Balch WE, Fox GE, Magrum LJ, Woese CR, Wolfe RS. Methanogens: reevaluation of a unique biological group. Microbiol Rev 1979; 43:260-296. PubMed

15. Ashburner M, Ball CA, Blake JA, Botstein D, Butler $\mathrm{H}$, Cherry JM, Davis AP, Dolinski K, Dwight SS, Eppig JT, et al. Gene ontology: tool for the unification of biology. The Gene Ontology Consortium. Nat Genet 2000; 25:25-29. PubMed doi:10.1038/75556

16. Badger JH, Olsen GJ. CRITICA: coding region identification tool invoking comparative analysis. Mol Biol Evol 1999; 16:512-524. PubMed

17. Delcher AL, Harmon D, Kasif S, White O, Salzberg SL. Improved microbial gene identification with GLIMMER. Nucleic Acids Res 1999; 27:4636-4641. PubMed doi:10.1093/nar/27.23.4636

18. Liolios K, Mavromatis K, Tavernarakis N, Kyrpides NC. The Genomes OnLine Database (GOLD) in 2007: status of genomic and metagenomic projects and their associated metadata. Nucleic Acids Res 2008; 36:D475-D479. PubMed doi:10.1093/nar/gkm884

19. Ewing B, Green P. Basecalling of automated sequencer traces using phred. II. Error probabilities. Genome Res 1998; 8:186-194. PubMed

20. Ewing B, Hillier L, Wendl M, Green P. Basecalling of automated sequencer traces using phred. I. Accuracy assessment. Genome Res 1998; 8:175185. PubMed

21. Anderson I, Ulrich LE, Lupa B, Susanti D, Porat I, Hooper SD, Lykidis A, Sieprawska-Lupa M, Dharmarajan L, Goltsman E, et al. Genomic characterization of Methanomicrobiales reveals three classes of methanogens. PLOS ONE 2009; 4:e5797. PubMed

doi:10.1371/journal.pone.0005797

22. Pati A, Ivanova N, Mikhailova N, Ovchinikova G, Hooper SD, Lykidis A, Kyrpides NC. GenePRIMP: A Gene Prediction Improvement Pipeline for microbial genomes. (Submitted) 2009.

23. UniProt Consortium. The Universal Protein Resource (UniProt) 2009. Nucleic Acids Res 2009; 37(Database issue):D169-D174. PubMed doi:10.1093/nar/gkn664

24. Haft DH, Selengut JD, White O. The TIGRFAMs database of protein families. Nucleic Acids Res 2003; 31:371-373. PubMed doi:10.1093/nar/gkg128

25. Finn RD, Tate J, Mistry J, Coggill PC, Sammut SJ, Hotz HR, Ceric G, Forslund K, Eddy SR, Sonnhammer EL, Bateman A. The Pfam protein families database. Nucleic Acids Res 2008; 36(Database issue):D281-D288. PubMed doi:10.1093/nar/gkm960

26. Claudel-Renard C, Chevalet C, Faraut T, Kahn D. Enzyme-specific profiles for genome annotation: PRIAM. Nucleic Acids Res 2003; 31:6633-6639. PubMed doi:10.1093/nar/gkg847

27. Kanehisa M, Araki M, Goto S, Hattori M, Hirakawa M, Itoh M, Katayama T, Kawashima S, Okuda S, Tokimatsu T, Yamanishi Y. KEGG for linking genomes to life and the environment. Nucleic Acids Res 2008; 36(Database issue):D480-D484. PubMed doi:10.1093/nar/gkm882

28. Tatusov RL, Fedorova ND, Jackson JD, Jacobs AR, Kiryutin B, Koonin EV, Krylov DM, Mazumder R, Mekhedov SL, Nikolskaya AN, et al. The COG database: an updated version includes eukaryotes. BMC Bioinformatics 2003; 4:41. PubMed doi:10.1186/1471-2105-4-41

29. Hunter S, Apweiler R, Attwood TK, Bairoch A, Bateman A, Binns D, Bork P, Das U, Daugherty L, Duquenne $L$, et al. InterPro: the integrative protein signature database. Nucleic Acids Res 2009; 37(Database issue):D211-D215. PubMed doi:10.1093/nar/gkn785

30. Emanuelsson O, Brunak S, von Heijne G, Nielsen $\mathrm{H}$. Locating proteins in the cell using TargetP, SignalP and related tools. Nat Protoc 2007; 2:953-971. PubMed doi:10.1038/nprot.2007.131

31. Krogh A, Larsson B, von Heijne G, Sonnhammer EL. Predicting transmembrane protein topology with a hidden Markov model: application to complete genomes. J Mol Biol 2001; 305:567580. PubMed doi:10.1006/jmbi.2000.4315 
32. Bland C, Ramsey TL, Sabree F, Lowe M, Brown K, Kyrpides NC, Hugenholtz P. CRISPR recognition tool (CRT): a tool for automatic detection of clustered regularly interspaced palindromic repeats. BMC Bioinformatics 2007; 8:209. PubMed doi:10.1186/1471-2105-8-209

33. Lowe TM, Eddy SR. tRNAscan-SE: a program for improved detection of transfer RNA genes in genomic sequence. Nucleic Acids Res 1997;

25:955-964. PubMed doi:10.1093/nar/25.5.955
34. Markowitz VM, Mavromatis K, Ivanova NN, Chen IMA, Chu K, Kyrpides NC. IMG ER: a system for microbial genome annotation expert review and curation. Bioinformatics 2009; 25:2271-2278. PubMed doi:10.1093/bioinformatics/btp393

35. Thauer RK, Kaster AK, Seedorf H, Buckel W, Hedderich R. Methanogenic archaea: ecologically relevant differences in energy conservation. Nat Rev Microbiol 2008; 6:579-591. PubMed doi:10.1038/nrmicro1931 